\title{
Spectrographic Equipment of the 200-inch Telescope
}

Previous to the designing of the $f / 0 \cdot 36$ Dr. G. E. Hale's article (see special supplement to Nature of February 8) observations made at Mount Wilson Observatory by means of the f/0.59 Rayton lens had shown that the apparent velocities of recession of the more remote nebulæ are extraordinarily high and that they increase with the distances of the nebulæ. These observations had been extended as far into space as appeared to be practicable with the $f / 0.59$ Rayton lens, and it was with the object of increasing the range within which the nebulæ could be investigated spectrographically that the designing of a spectrographic lens of larger aperture was undertaken by the British Scientific Instrument Research Association.

The equivalent numerical aperture $(0.85)$ of the Rayton lens was already very nearly as large as could be obtained in any well-corrected 'dry' lens, and it was obvious that an 'immersion' lens would be needed if any large increase of aperture were to be achieved. With an object-glass designed to be used with a fluid of chosen refractive index between the last lens and the photographic film or plate, the limit of numerical aperture theoretically obtainable is equal to the refractive index $(1 \cdot 55)$ of the gelatine of the photographic emulsion. This limit cannot be reached in practice, but it appeared probable that a numerical aperture of 1.4 might be obtainable, which would represent approximately a three-fold increase in 'rapidity', very nearly doubling the range within which the apparent velocities of the nebulæ could be investigated. On general grounds it was considered worth while to aim at a design which would give a flat field over the range of spectrum which is of main interest in studying nebular velocities, in order that difficulties associated with the use of curved films might be avoided. A preliminary exploration of possible designs indicated that a system to fulfil these requirements should be capable of being produced without great difficulty, and, out of the designs considered, one was selected for detailed computation.

It was foreseen that difficulties might arise in the subsequent use of the lens if the immersion fluid to be used with it should prove to have any deleterious effect on the usual photographic emulsions. Experimental tests on plates which had been immersed for several days in the selected immersion fluid showed that this liquid had no perceptible action on the plates, and that the liquid could be removed quickly and completely in readiness for the plates to be developed. As a precautionary measure the lens was designed so that, if necessary, the thickness of the back lens could be reduced sufficiently for the lens to be used with specially thin plates with their glass sides in immersion contact with the back lens; it was, however, made up to be used in direct im. mersion contact with the coated sides of ordinary photographic plates. In the work which has been done with the lens, there has been no evidence of action between the immersion fluid and the emulsion.

A lens designed for spectrographic work may be considered as distinct from all the usual types of object-glasses. It is required to form separate line-images from a series of beams of parallel light of different colours all travelling along directions inclined at different angles to the axis of the lens. The corrections required for a lens to be used in this way are unlike those necessary in photographic lenses, telescope object-glasses, etc., and can be obtained by somewhat different methods. Achromatism, for example, as the term is ordinarily used, is not essential, and a certain amount of astigmatism can be tolerated in the images formed on either side of the axis. A lens entirely free from secondary spectrum and with an anastigmatically flattened field would, it is true, be ideal for use as an astrospectrographic object-glass, but in view of the impossibility of producing such a lens with an adequate aperture for the problem in hand, alternative methods of obtaining the necessary types of corrections were investigated.

An ordinary achromatic object-glass, corrected so as to give an anastigmatically flattened field, would be of little value as a spectrographic objectglass except with curved films. Its secondary chromatic errors would cause light of different colours to be brought to focus in different planes, so that the surface passing through the images formed by light of different colours would be curved. An achromatic object-glass having a curved field when used as an ordinary lens could, however, give an accurately focused spectrum on a flat plate, provided that the curvature of field were exactly compensated by the variation of focal distance due to the residual (secondary) chromatic errors of the lens. In designing a lens of numerical aperture $1 \cdot 4$, it is necessary to follow the general lines adopted in the designing of 
microscope object-glasses of large aperture. The residual chromatic errors of such lenses are small, and the field-curvature is so large that it would more than compensate for the curvature which these residual chromatic errors would introduce in the focal surface of a well-dispersed spectrum. It is permissible, however, to ignore any slight astigmatism of a spectrographic object-glass, provided that the tangential astigmatic images of the spectrum lines are brought to focus on the photographic film or plate. Thus, it is permissible to compensate for the curvature of field left unbalanced by the 'secondary spectrum correction', by introducing corrections which would flatten the focal surface passing through the tangential astigmatic images.

The method used to flatten the tangential astigmatic field was one which, in addition, introduced under-correction for spherical aberration in oblique image-forming rays. For the complete system under consideration, comprising the collimator, prisms, and the $f / 0 \cdot 36$ lens, the obliquity of the ray-systems on one side of the axis increases as the wave-length of the light becomes shorter, and the under-correction at increased obliquity can be made to balance the over-correction due to shortening of the wave-length. Hence, proceeding outwards from the centre of the field, correction for spherical aberration can be maintained over a considerable distance. On the other side of the axis the obliquity of the ray-systems increases with increase of wave-length, and spherical aberration is increased more rapidly than it would be if the field were not flattened. Since, however, the important range of spectrum to be investigated is confined to the shorter visible and near ultraviolet radiations, imperfect imaging of the yellow and red regions of the spectrum is of no importance, and satisfactory imaging over the required range can be ensured by designing the lens so that some selected blue or violet radiation would pass axially through it

The design of the lens followed the lines indicated by the considerations briefly outlined above. It was necessary, of course, to have particulars of the angular dispersion which would be given by the prism system to be used with the lens, and also to be informed of any residual errors of the collimator lens. The design as finally developed was for a lens of the required aperture, with full spherical correction and giving a flat field for dispersed parallel beams covering the range of wave-lengths between $3600 \mathrm{~A}$. and $5000 \mathrm{~A}$. In the design, also, special attention was paid to ensuring that the sine condition should be fulfilled and to eliminating any independent coma of a higher order, so that the entire spectrum should be free from coma.
The mounting of the back component of the lens system required special consideration to ensure that optical contact should be maintained between the back lens and the photographic plate during the longest exposures likely to be given. In view, also, of the very short 'depth of focus' of the lens, specially fine differential-screw focusing adjustments were provided and the plate-carrier was designed as an integral portion of the lens mounting. A sensitive tilting adjustment was also fitted to the plate carrier in order that the plane of the photographic plates could be brought into coincidence with the focal surface. Both these adjustments were provided with clamping systems so that, when once the lens and plate-carrier had been set in their proper relative positions, plates could be changed with the certainty that each plate would automatically be brought into the proper focal plane.

When the collimator, prism system, and large aperture lens had been constructed, the complete system was examined optically in the laboratories of the British Scientific Instrument Research Association, and was found to realise fully the expectations founded on the calculations made during the designing of the lens. By adopting a similar method of examination, it will be possible at any time to mount the complete outfit in a telescope, ready adjusted, so that loss of time in testing the adjustments by trial exposures on astronomical objects may be entirely avoided.

The complete spectrographic equipment was delivered to the California Institute of Technology in December 1934 and was tested on the 100-inch telescope at Mount Wilson after the necessary mountings had been completed. In the tests first carried out on the remote nebulæ, the results obtained were disappointing. Work on other astronomical objects showed, however, that the optical performance of the system is of the high order indicated by the calculations and by the tests made before dispatch, and that the disappointing results obtained on the nebulæ were due to fogging of the spectra by light from the night-sky at Mount Wilson. To a large extent this is scattered light from the towns in the San Gabriel valley; at Mount Palomar, where the new 200-inch telescope is to be erected, the night-sky is appreciably darker, and it is confidently hoped that, when the lens comes to be used there, it will fulfil the purpose for which it was originally designed; although a limit to the range within which the nebulæ can be explored may possibly be set by the natural brightness of the sky due to auroral light.

In the meantime, the lens is proving of very great value in investigating differences between 
the radial velocities of the individual stars in starclusters in the extra-galactic nebulæ, and a wide variety of other uses is foreshadowed for which the lens is peculiarly fitted owing to its extreme rapidity.

Dr. Hale, in a letter describing some of the results already obtained, refers to the lens as "a great success optically", and expresses his congratulations on "a truly remarkable achievement". It reflects great credit on the optical industry of Great Britain to have produced a lens having such outstanding possibilities.

\section{Matthew Boulton as Scientific Industrialist}

$\mathrm{T}^{\mathrm{H}}$ HE commemoration in January last of the bicentenary of the birth of James Watt naturally directed attention to the notable part taken in the development and application of the steam engine by his partner Matthew Boulton, and in his "James Watt, Craftsman and Engineer", Mr. H. W. Dickinson expresses regret that hitherto no separate biography of Boulton has been written (see Nature of January 25, p. 129).

The career of Boulton, of course, has not escaped the writers on Watt and the steam engine, and Smiles in particular dealt at considerable length with many of his activities. But the life of Boulton, relating to which we imagine there is much unexplored material, deserves still closer study, for in the history of the industrial progress of Great Britain during the latter half of the eighteenth century, there is no more engaging figure. He was a man of admirable character, broad-minded, generous, energetic and far-sighted, displaying many of the attributes of a statesman. Unlike many of his contemporaries in the field of industry, he started life with social advantages, and early in life was a man of means. But if, as he himself declared, he loved money-getting projects, he was still more concerned that nothing but the highest standard should be aimed at, whether it was in connexion with his own affairs or those of the nation. As a captain of industry he had no equal, and was alike at home whether dealing with his employees, his partners, privy councillors or prime ministers.

Boulton was the senior of Watt by more than seven years, having been born in Birmingham in September 1728. The two first met when he was nearly forty years of age, and was the head of the largest manufacturing concern in the country. His father had begun as a silver stamper and piercer, and when he died in 1759 he left his son a well-established business for the manufacture of trinkets, steel buttons, buckles and the like. In 1762, Boulton married Ann Robinson of Lichfield and with her came a fortune of $£ 28,000$. This accession of wealth, instead of diminishing his interest in trade, enabled him to launch out on new ventures and he proceeded at once to erect the famous Soho Manufactory about two miles north of Birmingham, having for his partner John Fothergill. When completed, the Manufactory could house six hundred workpeople, and by 1767

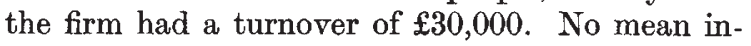
ventor himself, versed in the science of his day and possessing artistic tastes, Boulton introduced new machinery and processes, and was at as great pains as Wedgwood to apply art to industrial products. He had agents in many foreign capitals, his showroom in London was visited by dukes and lords, and at his house at Soho he dispensed a princely hospitality.

Like other manufacturers, one of the problems Boulton had to face was that of power supply, and one of the reasons for placing the factory at Soho was that water-power could be obtained from the Hockley Brook. The supply, however, was unreliable, and even before Watt first visited Birmingham, Boulton had already considered the proposal to instal a 'fire engine' for pumping the tail race water from the water wheels back to a reservoir. When Watt went to Soho in August 1768, Boulton was, therefore, ready to seize on any project which would effectively overcome his difficulties, and it is a remarkable tribute to his judgment of men and to his insight into the future of the steam engine that, although no patent had yet been secured and no full-sized engine erected embodying Watt's principle of the separate condenser, Boulton was ready at once to stake his all on the success of the new engine.

The patent was secured in January 1769 and a month later Boulton wrote to Watt that his "idea was to settle a manufactory near to my own by the side of our canal where I would erect all the conveniences necessary for the completion of engines, and from which manufactory we would serve all the world with engines of all sizes". Though in his short intercourse with Boulton he had conceived a great liking for him, to the nervous, desponding and impecunious Watt such a scheme might well have appeared rash, almost to madness, and as a matter of fact five valuable 